\title{
Análisis territorial de las condiciones de habitabilidad en el periurbano de la ciudad de Mar del Plata (Argentina), a partir de la construcción de un índice y de la aplicación de métodos de asociación espacial ${ }^{1}$
}

\author{
Laura Zulaica² y Juan Pablo Celemín²
}

\begin{abstract}
RESUMEN
El complejo y espontáneo proceso de conversión de tierras rurales en urbanas da como resultado la conformación de un territorio de interfase de alta vulnerabilidad socioambiental. El presente trabajo planteó como objetivo analizar en el territorio las condiciones de habitabilidad en el periurbano de Mar del Plata, a través de un índice que expresa la relación establecida entre el hábitat y el habitar. Por medio del empleo de patrones locales de identificación espacial se obtuvo una cartografía de clusters y otra de significancia, que permitieron descomponer el índice de autocorrelación espacial y ampliar la aproximación analítica del área de estudio. La aplicación de esta metodología reviste un gran interés en el estudio del periurbano ya que detecta zonas donde se evidencian contrastes socioambientales que afectan a la calidad de vida de la población.
\end{abstract}

Palabras clave: Periurbano, vulnerabilidad socioambiental, habitabilidad, calidad de vida.

\begin{abstract}
The complex and spontaneous conversion process of rural land in urban areas result in the formation of an interface area of high social and environmental vulnerability. This paper aimed to define living conditions in suburban Mar del Plata through an index that shows the relation between habitat and inhabit. The employment of local indicators of spatial autocorrelation resulted in two maps: cluster and significance maps that allowed the disintegration of the spatial autocorrelation index thus expanding the analytical approach of the study area. The application of this methodology is of great interest in the periurban studies since it detects areas where social end environmental contrasts affect the quality of life of the population.
\end{abstract}

Key words: Periurban, social and environmental vulnerability, habitability, quality of life.

1 Artículo recibido el 10 de julio de 2007 y aceptado el 6 de agosto de 2008.
2 Universidad Nacional de Mar del Plata, CONICET (Argentina).E-mail: Izulaica@ohns.ucsf.edu; ciam@mdp.edu.ar 
Los gobiernos locales enfrentan día a día crecientes desafíos para atender a la diversidad de problemáticas urbanas cada vez más complejas que caracterizan a las ciudades actuales. Armonizar los procesos de urbanización con la capacidad de brindar respuestas a los conflictos emergentes constituye un principio básico del desarrollo urbano sostenible.

La definición de desarrollo sostenible que se cita con mayor frecuencia es la propuesta por la Comisión de las Naciones Unidas sobre Medio Ambiente y Desarrollo, conocida también como Comisión Bruntland, de 1987. En su informe a la Asamblea General de las Naciones Unidas, titulado "Nuestro Futuro Común", la Comisión definió el desarrollo sostenible como "el desarrollo que satisface las necesidades del presente sin comprometer la capacidad de las generaciones futuras de satisfacer las propias" (Gallopín, 2003: 23).

En relación a este paradigma, Guimarães (2003: 30) enuncia y explica distintas dimensiones contenidas en el concepto de sustentabilidad que pueden sintetizarse de la siguiente forma: 1) una dimensión ecológica, que implica preservar la integridad de los procesos naturales que garantizan los flujos de energía y materiales en la biósfera y que, a la vez, preserva la biodiversidad en su sentido más amplio, objetivando la conservación de la dotación de los recursos naturales; 2) una dimensión social y cultural, que promueve el mantenimiento del sistema de valores, prácticas y símbolos de identidad, la igualdad y el bienestar humano; 3) una dimensión económica, tendiente a la gestión adecuada de los bienes ambientales congruente con las metas de la sustentabilidad ecológica y; 4) una dimensión política, que privilegia la complementariedad entre los mecanismos de mercado y la regulación pública promovida como política de Estado.

En términos semejantes y haciendo referencia específica a las ciudades, Fernández et al. (1999: 24) establece también cuatro dimensiones de la sustentabilidad urbana que conforman el paradigma del desarrollo urbano sostenible: sustentabilidad natural, sustentabilidad social, sustentabilidad eco- nómica y sustentabilidad política. Cada una de estas dimensiones o esferas propone una relectura de cuatro megatemas centrales en las políticas urbanas: la sustentabilidad (referida al capital natural), la habitabilidad, la productividad y la gobernabilidad, respectivamente. El mismo autor define la sustentabilidad ambiental como un punto de equilibrio de las cuatro manifestaciones sectoriales de políticas urbanas replanteadas en términos de sustentabilidad.

A partir de las conceptualizaciones anteriores, el presente trabajo propone un análisis territorial de las condiciones de habitabilidad, ligadas a la dimensión social de la sustentabilidad, en el área periurbana de la ciudad de Mar del Plata. En este sentido, la pérdida en las condiciones de habitabilidad corre paralela a la mayor insostenibilidad de los sistemas urbanos, considerando estos en un sentido amplio (Rueda, 1997: 1). Pero antes de definir los alcances del trabajo y las características del área de estudio, es pertinente realizar una revisión teórica de la noción de habitabilidad y de los conceptos asociados a este tema.

\section{La habitabilidad y su relación con la calidad de vida}

El concepto de habitabilidad tiene distintas acepciones; algunas de ellas se remiten exclusivamente al ámbito de la vivienda, mientras que otras exceden ese marco de análisis para hacer referencia a la satisfacción de las personas en un determinado escenario o grupo de escenarios. La habitabilidad desde esta última perspectiva es entendida como "la capacidad de los espacios construidos para satisfacer las necesidades objetivas y subjetivas de los individuos y grupos", es decir, involucra las esferas psíquicas y sociales de la existencia estable que podría equipararse a las cualidades ambientales que permiten el sano desarrollo físico, biológico, psicológico y social de la persona (Castro, 1999; citado por Landázuri Ortiz y Mercado Doménech, 2004: 90). De ahí su estrecha vinculación con el concepto de calidad de vida.

Velázquez (2001: 15) define a la calidad de vida como una medida de logro respecto 
de un nivel establecido como óptimo, teniendo en cuenta dimensiones socioeconómicas y ambientales dependientes de la escala de valores prevaleciente en la sociedad y que varían en función de las expectativas de progreso histórico. Entonces, este concepto se construye a partir de un "proceso" y es una aspiración que se determina como variable en el "tiempo". De allí que este término goce de un importante "dinamismo" y de ciertos niveles de subjetividad ${ }^{3}$.

Mejorar la habitabilidad de las ciudades supone incrementar la calidad de vida de sus habitantes y con ello la sustentabilidad social en su interior. En este sentido, Rueda (1997) plantea la habitabilidad urbana a partir de cuatro grandes categorías que se vinculan a la calidad de vida de los habitantes y por lo tanto, a la sustentabilidad de las ciudades: bienestar general de la persona, que implica su bienestar interno (espiritual y psicológico) y externo (su relación con el resto del conjunto social); bienestar ambiental, que refiere a la relación armónica con el entorno; bienestar psicosocial, que implica la satisfacción individual y; bienestar sociopolítico, que tiene que ver con participación social, seguridad personal y jurídica. De acuerdo a este enunciado, la habitabilidad constituye una adaptación entre las características de la situación real y las expectativas, capacidades y necesidades del individuo tal y como las percibe él y su grupo social (GIDES, 2003: 11).

De acuerdo con Moreno (2002: 3), la habitabilidad entendida como una meta de bienestar involucra, además del hecho físico de la vivienda, el ambiente sociocultural y el entorno. En el logro de la habitabilidad intervienen las cualidades físicas (ausencia o presencia de contaminación y deterioro, estado del paisaje desde el punto de vista estético, entre otras) tanto como las socioculturales (entramado social, redes de relaciones, imaginarios, pautas de consumo,

\footnotetext{
3 Existen numerosos conceptos en relación a la calidad de vida. Otros aportes pueden encontrarse en Abaleron (1998); García y Velázquez (1999); Olave Farías (2001); Velázquez y Gómez Lende (2005).
}

mecanismos de intercambio, tratamiento de los conflictos y seguridad, entre otras).

La relación existente entre habitabilidad, calidad de vida y sustentabilidad se enuncia en el Programa de las Naciones Unidad sobre Asentamientos Humanos (UN-HÁBITAT) al que adscribió Argentina. En dicho Programa se señala que la habitabilidad se vincula a las características y cualidades del espacio, entorno social y medio ambiente que contribuyen singularmente a dar a la gente una sensación de bienestar personal y colectivo e infunden la satisfacción de residir en un asentamiento determinado; las aspiraciones a la habitabilidad varían de un lugar a otro, cambian y evolucionan en el tiempo y difieren según las poblaciones que integran las comunidades.

\section{Hábitat y habitar, componentes de la habitabilidad}

A partir de las definiciones anteriores, las condiciones de habitabilidad urbana pueden ser analizadas a partir de dos componentes centrales implícitos: el hábitat y el habitar.

La noción de hábitat tiene sus raíces en la Ecología, no obstante ello, el reconocimiento de un hábitat humano asociado a las ciudades implica la aceptación de procesos e interacciones regidos por la cultura. De esta manera, el hábitat comprende lo relativo al sistema espacial y los recursos que elige un grupo para transitar por su existencia; que responde a las necesidades para desarrollar los procesos individuales y colectivos requeridos para realizar su vida productiva, laboral y doméstica en relación con un entorno mayor, y en intercambio con otros grupos de la sociedad. A su vez, está definido por el lugar geográfico donde se aloja, el cual tiene las condiciones de un espacio determinado y cualificado en concordancia con sus necesidades particulares y generales (CEHAP, 1998: 6).

Según Echeverría (2002), citado por Gordillo Bedoya (2005: 146), el hábitat es el lugar donde viven individuos, grupos humanos con una dimensión de la satisfacción 
relacionada con lo ambiental, lo sostenible, lo cultural y lo territorial. En la ciudad, como un espacio de relaciones económicas y sociales, la población realiza, entre otras, funciones de habitar. En ella se configura un mercado de bienes y servicios que ejerce presiones ambientales internas y externas (Moreno, 2002).

La noción de habitar ha sufrido una permanente redefinición semántica, acelerada en los momentos de profundo cambio cultural (Huber y Guérin, 2001: 2). El habitar varía en tiempo y espacio y conlleva diferentes modos de ocupación del territorio y, por lo tanto, distintas formas de vinculación con el entorno en donde se desenvuelve la vida de las personas.

A propósito de las distintas actividades sociales e individuales que derivan del habitar, el ser humano realiza una serie de contratos y códigos que le permiten existir en tanto sociedad más o menos organizada sobre parte de la superficie terrestre que ocupa; esto es un componente fundamental de la definición de territorio, decisivo en la regulación de las relaciones del ser humano con su entorno (Urruzola, 2006: 1).

Por su parte, Ben Altabef (2003: 3) resalta la idea de habitar que implica una acción o práctica social, inherente a la naturaleza humana, esto es el vivir, y también una cualidad en cuanto es generador de hábitos, es decir de usos y costumbres; esta práctica se desarrolla en un espacio, que genera un hábitat, ámbito de uso individual y colectivo del vivir, que es materia de especial interés desde el hacer cotidiano y que se ve condicionado y determinado por las diferentes modalidades del habitar.

Los conceptos de hábitat y habitar que guían el presente trabajo fueron tomados de Fernández et al. (1999: 274) y definidos anteriormente en Fernández et al. (1998), quienes caracterizaron el capital social de la ciudad de Mar del Plata a partir de indicadores sintéticos, estudio que constituye un marco de referencia para el análisis de la sustentabilidad social de la ciudad. En este sentido, el autor define al hábitat como el "subsistema de oferta" de recursos y servicios urbanos teóricamente disponibles res- pecto de una sociedad urbana determinada, pudiéndose hablar de una "naturaleza secundaria"; en tanto que el habitar es el "subsistema de demandas" de recursos y servicios urbanos teóricamente requeridos por una sociedad determinada, que comprende las condiciones aceptables (definidas por los hombres) para la vida social, incluyendo en este concepto la calidad de vida.

De lo mencionado anteriormente se deduce que los problemas de habitabilidad emergen en los términos de oferta y demanda entre el hábitat y el habitar, cuando los bienes, servicios y condiciones de la ciudad no brindan lo requerido para el habitar de la sociedad, siendo esas necesidades objetivas y subjetivas, dependientes estas últimas de las características culturales y del momento histórico por el cual se transita.

\section{El objeto de estudio}

Este estudio propone un análisis territorial de las condiciones de habitabilidad en el periurbano de la ciudad de Mar del Plata, Argentina, mediante la construcción de un índice sintético para luego aplicar métodos geoestadísticos a fin de establecer el grado de asociación espacial entre las unidades de análisis que conforman el periurbano. De esta manera, se espera encontrar áreas con valores altos del índice en cercanías de la ciudad y la costa, asociadas al valor paisajístico y turístico de esos lugares que favorece el desarrollo de segundas viviendas y la llegada de servicios básicos e infraestructura.

El índice se elaboró a partir de la selección de indicadores objetivos y cuantificables que expresan la relación entre el hábitat y el habitar. Se excluyeron del estudio indicadores subjetivos, sumamente importantes en análisis de las condiciones de habitabilidad pero muy difíciles de relevar y cuantificar, lo cual restringe el alcance de los resultados. En relación a ello y tomando como referencia las enunciaciones de Fernández et al. (1999: 280), es posible afirmar que una de las limitaciones del estudio de las condiciones de habitabilidad tiene que ver con el campo experimental donde se registra un desfasaje entre los numerosos aportes teóricos y la escasez de aportes ins- 
trumentales para mensurar y cualificar las evidencias empíricas.

Por su extensión y cantidad de habitantes, Mar del Plata constituye la tercera urbanización de la Provincia de Buenos Aires, después del Área Metropolitana de Buenos Aires y la ciudad de La Plata. De acuerdo al último censo elaborado por el Instituto $\mathrm{Na}$ cional de Estadísticas y Censos (INDEC, 2001) el Partido de General Pueyrredón registra en ese año un total de 564.056 habitantes de los cuales, el $95 \%$ son urbanos. Cuando se compara este valor con la población censada en 1991, se observa un crecimiento relativo del $5,9 \%$.

El incremento demográfico de Mar del Plata, cabecera del Partido de General Pueyrredón, ha respondido a procesos expansivos espontáneos que dieron como resultado la construcción de un territorio suburbano poco consolidado y de baja densidad de ocupación. Esta tendencia, restringida a las últimas décadas del siglo $\mathrm{XX}$, se hace evidente en el tercer escenario del proceso de poblamiento del Partido, descripto por Lucero (2004: 42). Según la autora, en este escenario, el ejido urbano de Mar del Plata se encuentra ocupado casi totalmente y bien consolidado, y el fenómeno de suburbanización prolonga el poblamiento más allá de los límites de la ciudad principal. La expansión sobre áreas periurbanas se ha dado de manera desordenada, con consecuencias negativas sobre el ambiente, la calidad de vida de la población y por lo tanto sobre las condiciones de habitabilidad.

Existen numerosas conceptualizaciones y revisiones teóricas para el abordaje del periurbano ${ }^{4}$. No obstante ello, los distintos autores concuerdan en que se trata de un territorio dinámico, susceptible a distintas transformaciones e intervenciones, frágil desde el punto de vista ambiental, desigual desde lo social y cuyas funciones son diversas (soporte de distintas actividades, fuente de recursos naturales, sumidero de desechos).

4 Adell (1999); Garay (1999); Morello (2000); Del Río (2002); Bozzano (2004); Di Pace (2004); Barsky (2005); entre otros.
El periurbano de Mar del Plata no escapa esta situación. Su conformación deriva del crecimiento de la ciudad, ha sido desordenada, sin planificación y guiada por los principales ejes de comunicación. Las consecuencias de este proceso se manifiestan en profundos contrastes territoriales expresados en las condiciones de habitabilidad de la población. En relación a esto, se observan claramente sectores que presentan condiciones mucho más favorables que otros, como es el caso del área costera vinculada al turismo.

\section{Área de estudio}

Considerando las cinco zonas periurbanas de Mar del Plata definidas en estudios previos (Ferraro y Zulaica, 2007; Zulaica y Ferraro, 2007), el presente trabajo toma como unidades de análisis a los radios censales establecidos por el INDEC que se corresponden con esas cinco zonas delimitadas. Las mismas fueron precisadas siguiendo los ejes de comunicación más importantes: eje Ruta 11 hacia el norte (Zona 1), Ruta 2 (Zona 2), Ruta 226 (Zona 3), Ruta 88 (Zona 4) y Ruta 11 hacia el sur (Zona 5).

Aunque la delimitación territorial es una arbitrariedad, no deja de ser una necesidad para poder acotar el área de estudio. El límite interno del periurbano (esto es, el borde urbano-periurbano) fue establecido a partir de la delimitación del ejido urbano de Mar del Plata especificada en el Código de Ordenamiento Territorial (COT) y la extensión de los servicios de saneamiento básicos (agua por red y cloacas), mientras que el límite externo (borde periurbano-rural) fue más difícil de determinar a partir de un criterio específico. El mismo conforma una franja difusa cuya extensión es variable dependiendo de cómo haya tenido lugar el proceso de expansión urbana sobre los principales ejes. En consecuencia, se asume que el grado de vinculación o conexión de los radios con dichos ejes definen la zona a la que pertenece cada radio.

El área así definida abarca unas 35.173 ha y, de acuerdo a los datos del último censo nacional, posee 130.056 habitantes distribuidos de la siguiente manera: un $9,9 \%$ en la Zona 1 ; un $10,3 \%$ en la Zona 2 ; 
un $35,9 \%$ en la zona 3 ; un $34,9 \%$ en la zona 4 y finalmente, el $8,8 \%$ restante en la Zona 5. La Figura $N^{0} 1$ muestra la localización del Partido de General Pueyrredón y del área de estudio, diferenciando las cinco zonas que componen el periurbano de Mar del Plata.

\section{Desarrollo metodológico}

\section{Construcción del índice}

El proceso metodológico de construcción de un índice que sintetiza las condiciones de habitabilidad $(\mathrm{ICH})$ en el periurbano presenta una gran complejidad dado que deben seleccionarse variables representativas de la situación que se pretende mostrar. Dichas variables no son directamente extrapolables a otros espacios ya que cada uno de ellos posee especificidades y dinámicas que le son propias. Por lo tanto, esta característica hace que la construcción metodológica varíe según los diferentes ámbitos geográficos. Es importante recordar que las variables son aquellos atributos, relaciones o contextos que se selec- cionan como relevantes para describir a las unidades de análisis. Consiguientemente, toda variable es un criterio de clasificación que se emplea para diferenciar las unidades de análisis. La integración de variables, o en bien una sola variable, definen indicadores. Estos proveen información agregada y sintética respecto a un fenómeno más allá de su capacidad de representación propia (Rueda, 1999: 11).

Un problema metodológico reside en el empleo de unidades territoriales artificiales establecidas administrativamente por organismos oficiales que no suelen reflejar necesariamente de modo estricto los fenómenos sociales y territoriales existentes. Dentro de la Geografía, y en el uso de Sistemas de Información Geográfica (SIG), se conoce a esta cuestión como el Problema de la Unidad Espacial Modificable (Moreno Jiménez, 2006: 6). Asimismo, el empleo de SIG ha sido de suma utilidad en la representación de los contrastes ya que mediante capas de información se da claridad a la interpretación de los procesos socioterritoriales y ambientales.

Figura $\mathrm{N}^{0} 1$

PARTIDO DE GENERAL PUEYRREDÓN: LOCALIZACIÓN DEL ÁREA DE ESTUDIO
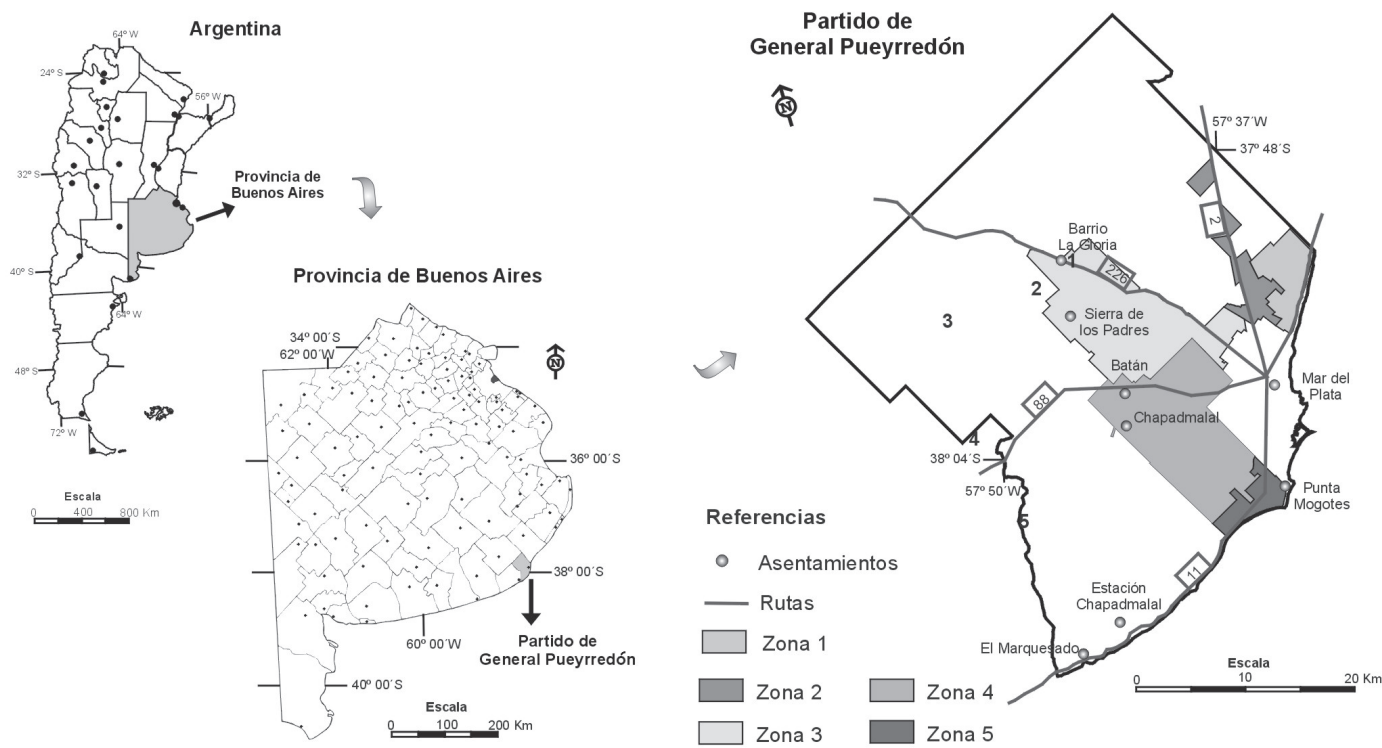

Fuente: Elaboración propia en base a Zulaica y Ferraro (2007). 
A los fines prácticos y de operacionalización de las condiciones de habitabilidad mediante un índice que muestre la relación establecida entre el hábitat y el habitar en cada unidad censal, se asume en el trabajo que el escenario deseable por parte de la sociedad, correspondiente al subsistema de demandas (habitar), coincide con los valores de condiciones más favorables alcanzados por las variables elegidas en el periurbano en estudio. El hábitat, está determinado entonces por la oferta de bienes, servicios y condiciones que existen realmente en la unidad censal.

\section{Selección de indicadores}

Con el fin de diferenciar las condiciones de habitabilidad, se elaboró el ICH a partir de la integración de siete indicadores que sintetizan las condiciones de habitabilidad en el periurbano. Los indicadores abarcan seis dimensiones y se enuncian en el Cuadro $N^{0} 1$. Cada indicador se encuentra representado por una o más variables obtenidas del último Censo Nacional (2001), que fueron ponderadas de manera directa, de acuerdo a su grado de importancia sobre las condiciones de habitabilidad ${ }^{5}$. En el caso de la dimensión ambiental, los datos fueron obtenidos de información proveniente del Municipio, imágenes satelitales y trabajo de campo. Asimismo, se indica si la variable es de beneficio (+) o de costo (-) y su rango porcentual registrado.

Las primeras tres dimensiones (salud y servicios esenciales, habitacional y accesibilidad) se relacionan con las problemáticas emergentes del periurbano e incluyen indicadores referentes a la cobertura de algunos servicios urbanos y a las condiciones de vida al interior de la vivienda. Las tres dimensiones restantes (educación, pobreza y dimensión ambiental) se refieren a las características de los grupos sociales y a su calidad de vida en relación con el entorno.

\footnotetext{
5 Gran parte de las dimensiones, indicadores y ponderaciones han sido desarrollados y utilizados en estudios previos para la construcción de índices de calidad de vida (Marinelli et al., 1999; Lucero et al., 2005; Riviere et al., 2005; Celemín, 2007; Mikkelsen, 2007; entre otros).
}

La calidad sanitaria está estrechamente ligada a la posibilidad de acceso a los servicios que el usuario necesite en el momento y lugar preciso, en suficiente cantidad y a un costo razonable $y$, particularmente, a la disponibilidad de servicios esenciales como son la provisión de agua de red y cloacas. El indicador relativo a la calidad sanitaria se encuentra desagregado en tres variables provenientes del último censo: "Porcentaje de población que no posee obra social o cobertura médica asistencial", "Porcentaje de hogares con inodoro con descarga de agua y desagüe a red pública" y "Porcentaje hogares con agua proveniente de la red pública".

La calidad de la vivienda es un indicador habitacional que se vincula directamente con las condiciones del hábitat. La vivienda representa un componente fundamental, en tanto entidad que no solamente es parte de las necesidades de subsistencia de una sociedad particular, sino que se constituye en un elemento más de inserción e identificación social. Se utilizaron dos variables: "Porcentaje de viviendas con calidad de materiales I (CALMAT I)", que comprende a aquellas compuestas por materiales resistentes y sólidos en todos los parámetros (pisos, paredes y techos) e incorpora todos los elementos de aislamiento y terminación y "Porcentaje de hogares con baño de uso exclusivo".

El indicador movilidad urbana se refiere a la posibilidad de la población para trasladarse desde su ámbito de residencia al núcleo urbano de Mar del Plata por medio del transporte público. La disponibilidad del mismo asegura mayor accesibilidad a servicios de salud, educación, culturales, recreativos, etc., influyendo positivamente sobre las condiciones de habitabilidad. La variable utilizada para este indicador es el "Porcentaje de hogares con existencia de transporte público a menos de $300 \mathrm{~m}^{\prime \prime}$ de acuerdo a la definición establecida en la base de usuarios del INDEC.

El nivel de instrucción, indicador de la dimensión educación, permite caracterizar la vulnerabilidad de los grupos sociales ya que presenta una estrecha vinculación con la inserción de la población al mercado laboral. En este caso, para evaluar el nivel de 
instrucción de la población del área de estudio, se tomaron las siguientes variables provistas por el INDEC "Porcentaje de población de 12 años o más con nivel de instrucción menor a primario completo". En contraposición, quienes culminan sus estudios terciarios y universitarios, retrasan el ingreso al segmento de población económicamente activa y tienen la posibilidad de acceder a una buena remuneración económica. Para este caso se seleccionó la variable "Porcentaje de población de 20 años o más con nivel de instrucción superior (terciario o universitario) completo".

Las Necesidades Básicas Insatisfechas (NBI) constituyen un indicador válido para captar la pobreza estructural ${ }^{6}$ en área determinada. Comprenden el primer grupo de indicadores introducido por la Comisión Económica para América Latina (CEPAL) a comienzos de los años ochenta para identificar carencias críticas de la población y caracterizar la pobreza (Feres y Mancero, 2001: 8).

Se considera pobre a un hogar o a las personas que en habitan en dicho hogar, cuando reúnen una o más de las siguientes condiciones: 1) Hacinamiento: hogares con más de tres personas por cuarto; 2) Vivienda: hogares que habitan en una vivienda de tipo inconveniente (pieza de inquilinato, pieza de hotel o pensión, casilla, local no construido para habitación o vivienda móvil, excluyendo casa, departamento y rancho); 3) Condiciones sanitarias: hogares que no tienen ningún tipo de retrete; 4) Asistencia escolar: hogares que tienen al menos un niño en edad escolar (6 a 12 años) que no asiste a la escuela y; 5) Capacidad de subsistencia: hogares que tienen cuatro o más personas que dependientes por cada miembro de la familia ocupado, cuyo jefe no haya completado el tercer grado de escolaridad primaria.

La dimensión ambiental considerada en el trabajo tiene que ver directamente con

\footnotetext{
6 Los "pobres estructurales" son aquellos que requieren de una importante inversión material o esfuerzo personal para superar el estado de precariedad social en la que se encuentran.
}

las condiciones del entorno sobre el cual se desarrolla la vida de las personas. Uno de los principales problemas ambientales que se registran en las ciudades es la acumulación de los residuos. Es por ello que la existencia de un servicio regular de recolección garantiza una menor presencia y dispersión de los mismos en la vía pública. La variable utilizada para dimensionar las condiciones de higiene urbana es el "Porcentaje de población que reside en viviendas con servicio regular de recolección de residuos", información también proveniente del último censo.

Asimismo, a la hora de evaluar las condiciones de habitabilidad, se tuvo en cuenta la exposición a impactos ambientales por actividades industriales, mineras y de disposición de residuos. Los efectos negativos que ocasionan sobre el ambiente las actividades señaladas son ampliamente conocidos, asociándose fundamentalmente a distintos tipos y grados de contaminación y degradación de recursos naturales. Se localizaron las zonas industriales del área de estudio (un distrito industrial y Parque Industrial), los lugares en los que se desarrolla la actividad minera y el sitio de disposición final de residuos sólidos urbanos. Los distritos industriales (I), establecidos por el Código de Ordenamiento Territorial, se corresponden con zonas destinadas a la localización de usos comerciales, de servicios, depósitos e industrias, cuya complejidad y especificidad funcional resultan inconvenientes para la residencia de alta y media densidad.

Los efectos nocivos de estas actividades se extienden generalmente más allá de su localización puntual, es por ello que se creó un buffer alrededor de las mismas permitiendo establecer la variable "Porcentaje de la superficie del radio censal a menos de 501 metros de actividades industriales, mineras y de disposición de residuos". La definición del área de influencia de los impactos es de carácter intuitivo y se basa en el conocimiento de la zona que permite determinar hasta dónde se verifican efectos observables de las actividades sobre el medio, criterios ampliamente desarrollados en Conesa Fernández-Vítora (1997), Canter (1998), Gómez Orea (1999) y Echechuri et al. (2002). 
de la ciudad de Mar del Plata (Argentina), a partir de la construcción

DE UN ÍNDICE Y DE LA APLICACIÓN DE MÉTODOS DE ASOCIACIÓN ESPACIAL

Cuadro $\mathrm{N}^{\circ} 1$

DIMENSIONES, INDICADORES, VARIABLES Y PONDERACIONES UTILIZADAS EN ICH

\begin{tabular}{|c|c|c|c|c|}
\hline Dimensión & Indicador & Variables & $\begin{array}{l}\text { Ponde- } \\
\text { ración }\end{array}$ & $\begin{array}{c}\text { Rango } \\
(\%)\end{array}$ \\
\hline $\begin{array}{l}\text { Salud y } \\
\text { servicios } \\
\text { esenciales }\end{array}$ & Calidad sanitaria & $\begin{array}{l}\text { - Porcentaje de población que } \\
\text { posee obra social o cobertura } \\
\text { médica asistencial. (+) } \\
\text { - Porcentaje de hogares con } \\
\text { inodoro con descarga de agua } \\
\text { y desagüe a red pública. (+) } \\
\text { - Porcentaje hogares con agua } \\
\text { proveniente de la red pública. (+) }\end{array}$ & 10,0 & $\begin{array}{l}3,7-84,2 \\
0-99,64\end{array}$ \\
\hline $\begin{array}{l}\text { Habita- } \\
\text { cional }\end{array}$ & $\begin{array}{l}\text { Calidad de } \\
\text { la vivienda }\end{array}$ & $\begin{array}{l}\text { - Porcentaje hogares con uso } \\
\text { exclusivo de baño. (+) } \\
\text { - Porcentaje de hogares que } \\
\text { presentan calidad de los materiales } \\
\text { I definida por el INDEC. (+) }\end{array}$ & $\begin{array}{l}7,5 \\
7,5\end{array}$ & $\begin{array}{l}66,67-100 \\
2,6-98,6\end{array}$ \\
\hline $\begin{array}{l}\text { Accesi- } \\
\text { bilidad }\end{array}$ & Movilidad urbana & $\begin{array}{l}\text { - Porcentaje de hogares con } \\
\text { existencia de transporte público } \\
\text { a menos de } 300 \mathrm{~m} .(+)\end{array}$ & 10,0 & $0-100$ \\
\hline Educación & Nivel de instrucción & $\begin{array}{l}\text { - Porcentaje de población de } \\
12 \text { años o más con nivel de } \\
\text { instrucción menor a primario } \\
\text { completo. (-) } \\
\text { - Porcentaje de población de } \\
20 \text { años o más con nivel de } \\
\text { instrucción superior (terciario } \\
\text { o universitario) completo. (+) }\end{array}$ & 7,5 & $13,5-92,9$ \\
\hline Pobreza & $\begin{array}{l}\text { Necesidades Básicas } \\
\text { Insatisfechas }\end{array}$ & $\begin{array}{l}\text { - Porcentaje de hogares con } \\
\text { Necesidades Básicas } \\
\text { Insatisfechas (NBI). (-) }\end{array}$ & 15,0 & $13,9-100$ \\
\hline \multirow[t]{2}{*}{ Ambiental } & Higiene urbana & $\begin{array}{l}\text { - Porcentaje de hogares con } \\
\text { existencia de servicio regular } \\
\text { de recolección de residuos. (+) }\end{array}$ & 5,0 & $0-100$ \\
\hline & $\begin{array}{l}\text { Exposición a } \\
\text { impactos } \\
\text { ambientales de } \\
\text { actividades } \\
\text { industriales, mineras } \\
\text { y de disposición } \\
\text { de residuos }\end{array}$ & $\begin{array}{l}\text { - Porcentaje de la superficie del } \\
\text { radio censal a menos de } 501 \\
\text { metros de actividades industriales, } \\
\text { mineras y de saneamiento urbano } \\
\text { (disposición de residuos y planta } \\
\text { de tratamiento de efluentes } \\
\text { cloacales). (-) }\end{array}$ & 20,0 & $0-100$ \\
\hline Total & 100,0 & $0-100$ & & \\
\hline
\end{tabular}

Fuente: Elaboración propia. 


\section{Procedimiento}

Los valores para cada variable y unidad espacial fueron estandarizados de acuerdo a las fórmulas matemáticas que se enuncian a continuación y su sentido positivo o negativo. De esta manera se pueden compatibilizar variables de costo y beneficio sin que se anulen mutuamente:

- Variables cuyo incremento implica peor situación relativa. Variables de costo (-):

$$
P E_{i}=\left(\frac{M-x_{i}}{M-m}\right) * 100
$$

- Variables cuyo incremento implica mejor situación relativa. Variables de beneficio $(+)$ :

$$
P E_{i}=1-\left(\frac{M-x_{i}}{M-m}\right) * 100
$$

Siendo $\mathrm{PE}_{\mathrm{i}}=$ puntaje estándar de i-ésimo dato, $x_{i}=$ el dato original a ser estandarizado, $M=$ mayor valor de la variable, $\mathrm{m}=\mathrm{me}$ nor valor de la variable.

El ICH consiste en la sumatoria de los valores índice de cada variable ponderados según el peso relativo estipulado. El resultado reviste un valor teórico donde el rango entre 0 y 100 refleja la peor y mejor situación, respectivamente.

\section{Autocorrelación espacial y patrones locales de identificación espacial (LISA)}

La utilidad de la autocorrelación espacial se encuentra en su capacidad para estudiar la forma en que un fenómeno se propaga a través de las unidades espaciales y si tal comportamiento corresponde a algún modelo de difusión conocido o bien para estudiar la segregación espacial de alguna característica. En definitiva, refleja el grado en que objetos o actividades en una unidad geográfica son similares a los objetos o actividades en unidades geográficas próximas (Vilalta y Perdomo, 2005: 326).

El concepto de autocorrelación espacial parte del principio de Tobler que plantea que en el espacio geográfico todo se encuentra relacionado con todo, pero los espacios más cercanos están más relacionados entre sí. Por lo tanto, se intenta medir la correlación que una misma variable tiene en diferentes unidades espaciales contiguas en una perspectiva horizontal dando lugar a una de estas tres posibilidades:

- Autocorrelación espacial positiva: las unidades espaciales vecinas presentan valores próximos. Indica una tendencia al agrupamiento de las unidades espaciales.

- Autocorrelación espacial negativa: las unidades espaciales vecinas presentan valores muy disímiles. Indica una tendencia a la dispersión de las unidades espaciales.

- Sin autocorrelación: no ocurre ninguna de las dos situaciones anteriores. Por lo tanto, los valores de las unidades espaciales vecinas presentan valores producidos en forma aleatoria.

La autocorrelación espacial puede ser univariada o bivariada. En un gráfico de dispersión, en el eje $x$ aparecen los valores estandarizados de una variable para cada unidad espacial y en el y se encuentran los valores estandarizados del promedio de los valores de las unidades espaciales vecinas para la misma variable (en el caso de la autocorrelación espacial univariada) o de una segunda variable (autocorrelación espacial bivariada). En ambos casos, la recta de regresión lineal muestra el grado de asociación entre la variable y los valores contiguos de la misma u otra variable considerada.

El índice I de Moran es el cálculo más antiguo -data de 1950- y es típica su utilización en la detección y medición de la autocorrelación espacial comparando los valores de cada localización con los valores de las localizaciones vecinas. Los resultados de este índice varían entre el -1 y el 1 , representando las correlaciones mínimas (máxima dispersión) y máximas (máxima concentración) respectivamente, y donde el cero significa un patrón espacial totalmente aleatorio.

Un análisis más detallado de la autocorrelación espacial se realiza a través del reconocimiento de patrones locales de identificación espacial (LISA). Este método 
de la ciudad de Mar del Plata (Argentina), a partir de la construcción

DE UN ÍNDICE Y DE LA APLICACIÓN DE MÉTODOS DE ASOCIACIÓN ESPACIAL

descompone el índice I de Moran y verifica en cuánto contribuye cada unidad espacial a la formación del valor general, permitiendo obtener un valor de significancia para cada cluster formado por los valores similares de cada unidad espacial y sus vecinos. Además, la aplicación del método LISA entre todas las unidades espaciales genera valores proporcionales al indicador global. Como resultado se obtienen dos mapas. El primero es el conocido como cluster o de agrupamiento, donde cada unidad espacial se diferencia de acuerdo al tipo de autocorrelación espacial que posee en relación a sus unidades espaciales vecinas. El segundo indica el nivel de significancia que presenta a las unidades con I de Moran relevantes en base a su relación de contigüidad a partir de un procedimiento de aleatorización que muestra para cada unidad espacial la proba- bilidad de que sus relaciones de contigüidad se produzcan de manera aleatoria (Buzai y Baxendale, 2006: 366).

Para obtener los mapas correspondientes a esta sección se recurrió al software GeoDa de acceso gratuito cuya principal potencialidad radica en el análisis de datos espaciales (Anselin, 2004).

\section{Resultados y discusión}

La integración de los indicadores de condiciones del hábitat y del habitar en un único índice de habitabilidad siguiendo el procedimiento explicado en el desarrollo metodológico, permitió arribar a la representación cartográfica que se muestra en la Figura $\mathrm{N}^{\circ} 2$.

Figura $\mathrm{N}^{\circ} 2$

ÍNDICE DE CONDICIONES DE HABITABILIDAD

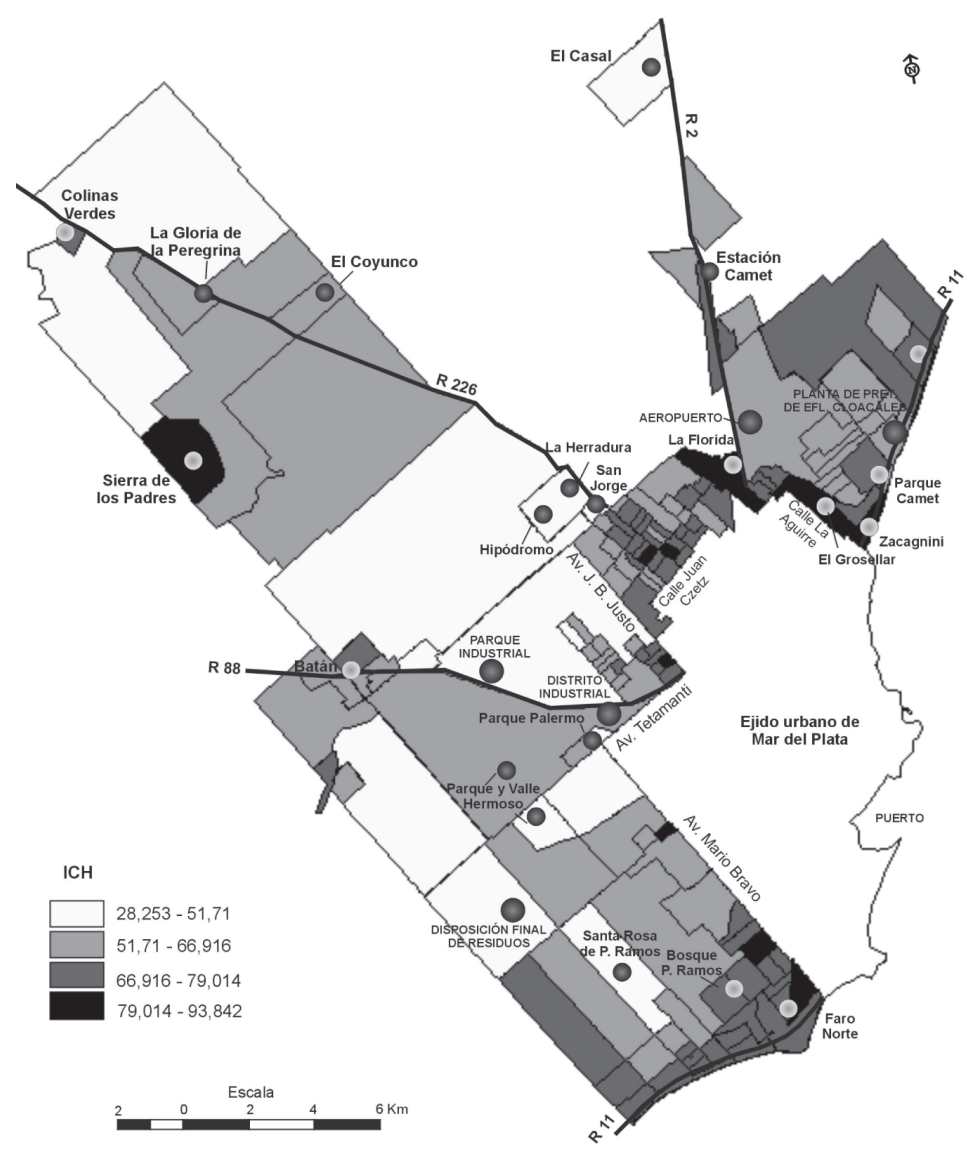

Fuente: Elaboración propia. 
Las mejores condiciones de habitabilidad estimadas a partir del cálculo del índice, se presentan fundamentalmente en los siguientes sectores del periurbano de Mar del Plata:

- Áreas colindantes con el límite norte del ejido urbano (sectores de los barrios La Florida, El Grosellar, Zacagnini, entre otros) en Zonas 1 y 2.

- Áreas situadas en las proximidades del límite urbano-periurbano oeste en Zona 3.

- Áreas periurbanas de Sierra de los Padres en Zona 3.

- Área costera del sector sur (Barrio Faro Norte) en Zona 5.

Dichas áreas exhiben los valores más altos del $\mathrm{ICH}$ que se ubican entre 80 y 94 puntos. Abarcan 970 ha, que corresponden al 2,8\% de la superficie total del periurbano y agrupan 15.219 habitantes, es decir un $11,7 \%$ de la población que vive en la interfase.

Las condiciones favorables se localizan sobre los ejes costeros norte (Zona 1) y especialmente en el sur (Zona 5). Las zonas citadas manifiestan una baja dinámica poblacional (Sagua, 2004: 162) y constituyen áreas de segundas residencias o residencias de veraneo asociadas al potencial turístico de las playas. Este hecho es mucho más significativo sobre el eje de la Ruta 11 hacia el sur (Zona 5) que sobre la misma ruta en sentido opuesto debido a que en este último, las playas alcanzan menor extensión, existiendo barrancos costeros sometidos a crecientes procesos de erosión. Asimismo, en este sector se localiza la Planta de Pretratamiento de efluentes cloacales de Mar del Plata, que arroja con escaso nivel de tratamiento los desechos al mar, situación que ocasiona una desvalorización de terrenos circundantes.

El eje de la Ruta 11 hacia el sur conforma el eje litoral denominado "Corredor Sur" (Burmester et al., 2003: 27). El área presenta un alto potencial paisajístico y amplia dotación de infraestructura y servicios turísticos (complejos privados, balnearios, viviendas secundarias y de temporada, comercios, etc.) que aprovechan la extensión de las playas, acantilados y el amplio frente marítimo forestado. En esa misma zona se presenta el Barrio Peralta Ramos en el que vive población de carácter permanente con alto poder adquisitivo. Dicho barrio se destaca por la extensión del bosque implantado y la calidad de las viviendas. En el mismo sector costero pero hacia el interior, sobre la Avenida Jorge Newbery, se destacan áreas de alto dinamismo en las cuales se están construyendo barrios privados sobre grandes extensiones de terrenos.

En las Zonas 2 y 3, en áreas próximas al ejido urbano, y cercanas al eje de la Ruta 2, se encuentran asentamientos de sectores con ingresos medios y altos en barrios que si bien no son cerrados, algunos poseen seguridad y se destacan por su amplio parquizado y forestación. El citado eje constituye el principal medio de enlace del municipio con el Área Metropolitana de Buenos Aires y posee la mayor intensidad de flujos respecto del resto de los ejes. A pesar de iniciarse su entorno como ámbito de residencias secundarias, se manifiesta actualmente una clara tendencia a la vivienda permanente, condición favorecida por la accesibilidad y la extensión de servicios urbanos.

En sectores aledaños al eje de la Ruta 226 (Zona 3), se localiza una villa serrana (Sierra de los Padres) de alto dinamismo, habitada por población en general de altos ingresos que se instalan motivados por el potencial paisajístico de la sierra bordeada por cultivos intensivos y por la búsqueda de tranquilidad fuera del ejido de la ciudad.

Las áreas con extremas condiciones de habitabilidad ( $\mathrm{ICH}$ inferiores a 45) se distribuyen en una superficie más extensa (7.874,8 ha, 22,4\% del total) y agrupan menor población (4.600 habitantes, 3,5\% del total). Estas últimas se localizan en áreas más alejadas de la planta urbana e incluyen:

- Sectores de los barrios Santa Rosa del Mar de Peralta Ramos, Parque Hermoso y Valle Hermoso, Parque Palermo y sectores más retirados situados al sur de la planta urbana (Zona 4). 
- Barrios San Jorge, Hipódromo y La Herradura y un área periurbana situada al sur de la Ruta 226 que presenta amplio desarrollo de agricultura intensiva (Zona 3).

- Asentamiento El Casal, situado sobre el eje de la Ruta 2 (Zona 2).

Como puede observarse en la Figura $\mathrm{N}^{\circ}$ 2, las condiciones más bajas de habitabilidad se presentan en áreas de las Zonas 3 y 4. La Ruta 226 presenta situaciones críticas en barrios próximos al ejido urbano, los cuales conforman asentamientos espontáneos y sumamente precarios con severos conflictos sociales y problemas con el régimen de tenencia de la tierra (ocupación en tierras fiscales o privadas). También se destacan asentamientos dispersos vinculados a la agricultura intensiva (horticultura), Ilevada a cabo en su mayor parte por migrantes de países limítrofes, población vulnerable que desarrolla la actividad en condiciones de alta precariedad y que se halla expuesta permanentemente a diversas situaciones de riesgos, en especial derivados del uso inade-

Figura $\mathrm{N}^{\circ} 3$ MAPA CLUSTER

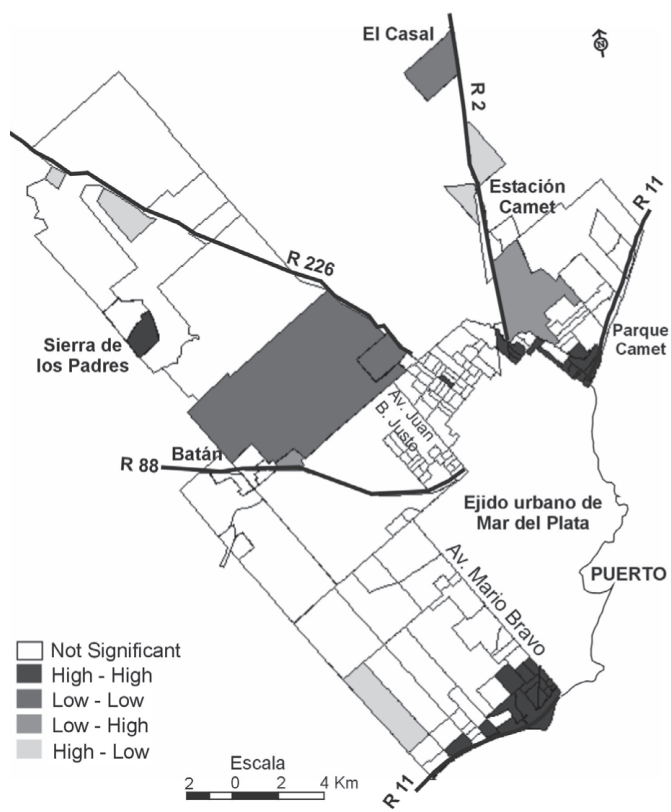

Fuente: Elaboración propia. cuado de agroquímicos. Esta problemática ha sido estudiada y documentada por Burmester (2004).

El eje de la Ruta 88 (Zona 4) que conecta la ciudad de Mar del Plata con la localidad de Batán y otros municipios del sur bonaerense, se lo reconoce como una zona industrial. Allí se localizan el Parque Industrial y actividades extractivas mineras como canteras y hornos de ladrillos. En esta dirección se han dado radicaciones de población mediante sistemas de condominio de parcelas rurales, intervenciones que generan suelo urbano de bajo precio, adquirido por grupos de escasos recursos (Bonavena, 2002). Asimismo, se ubicaron diferentes sitios de disposición final de residuos provenientes de Mar del Plata, depositados sin tratamiento y a cielo abierto. En las inmediaciones del actual predio se presentan asentamientos de extrema precariedad que viven de "la basura" (Riviere y Celemín, 2006). Sobre las avenidas Mario Bravo y Tetamanti, se han instalado asentamientos que se destacan por su condición de precariedad y alta vulnerabilidad socioambiental.
Figura $\mathrm{N}^{\circ} 4$ MAPA DE SIGNIFICANCIA

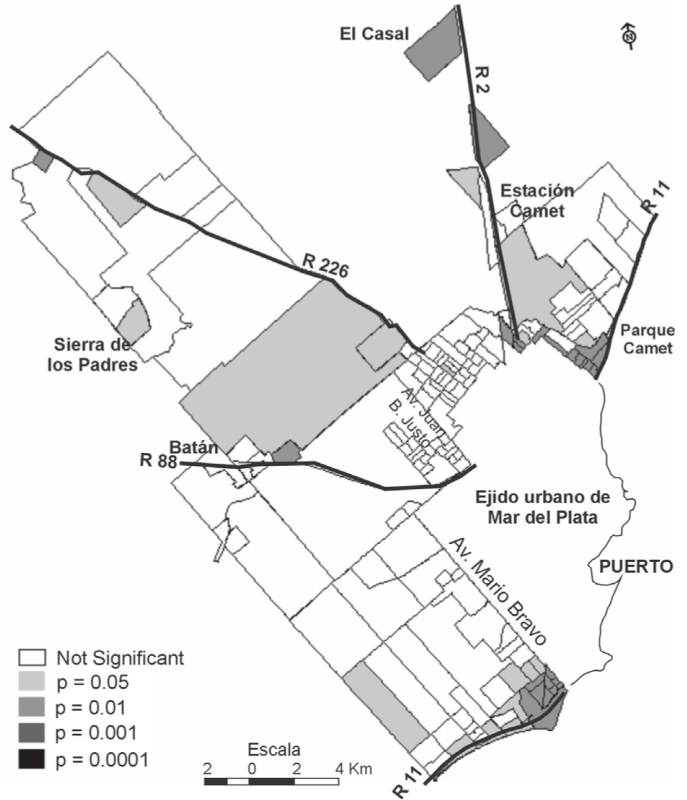

Fuente: Elaboración propia. 
La aplicación de la autocorrelación espacial produjo como resultado una I de Moran de 0.4252 , es decir un valor positivo mediobajo. Asimismo, a partir del empleo de LISA se obtuvo una ampliación destacable de la aproximación analítica por medio de la elaboración de dos nuevos productos cartográficos: el mapa de clusters o agrupamientos (Figura $N^{\circ} 3$ ) y el mapa de significancia que descomponen el índice de autocorrelación (Figura $\mathrm{N}^{\circ} 4$ ).

Ambos mapas enseñan coincidencias espaciales en sus resultados ya que los radios costeros de la Zona 5 y los limítrofes con la ciudad de Mar del Plata de las Zonas 1 y 2 son aquellos que presentan un cluster de agrupación alto-alto (high-high). Asimismo, son los mismos que presentan un valor $p$ de 0,001 , es decir, que solo un caso cada 1.000 respondería a una configuración espacial aleatoria. Por otra parte, un radio extenso de la Zona 3 responde al cluster bajobajo (low-low) con un nivel de significancia del $5 \%$. El resto de las unidades consideradas responde a situaciones mixtas (high-low, low-high) y la mayoría presenta valores no significativos, representados con el color blanco que se establece, por ahora, en lugares de mezcla en las condiciones evaluadas a través del índice, sin un patrón definido de vecindad positiva o negativa en la similitud de sus características (Lucero y Celemín, 2008: 100).

\section{Consideraciones finales}

El análisis territorial de las condiciones de habitabilidad a partir de la relación establecida entre el hábitat y el habitar, muestra que el periurbano dista mucho de aproximarse a los logros de equidad y bienestar social acordes con los principios de sustentabilidad, que solo se manifiestan en escasos sectores de la población.

La diversidad de situaciones identificadas a partir del $\mathrm{ICH}$, pone en evidencia la heterogeneidad socioterritorial y ambiental que caracteriza al periurbano. Si bien la conformación de este espacio responde a procesos que se dan en la ciudad, su abordaje como una unidad independiente acentúa las diferenciaciones internas de estas áreas en expansión. Dicho abordaje implicó efectuar "un recorte" de la realidad que permitió identificar importantes contrastes territoriales entre sectores con buenas condiciones de habitabilidad (costeros, Sierra de los Padres, barrios situados al norte cercanos al ejido urbano) y zonas con severas problemáticas socioambientales en casi todos los espacios restantes.

La distribución de las condiciones de habitabilidad en el periurbano responde a una dinámica propia y diferente a la presente en los espacios urbanos y rurales pero que no puede ser entendida sin estos últimos. En este sentido, las metodologías de construcción de índices asumen ciertos niveles de abstracción ya que su capacidad de síntesis excede la explicación de los procesos que tienen lugar en dichas áreas, por lo que se constituyen en una primera aproximación en el estudio del tema. No obstante ello, aparecen como herramientas válidas para estudiar la distribución espacial de las variables seleccionadas y visualizar rápidamente la relación entre el hábitat y el habitar en cada unidad de análisis.

En relación a las variables e indicadores analizados, se asume que los mismos son restringidos para definir las condiciones de habitabilidad en especial porque no incorporan aspectos subjetivos del habitar. A partir de esta consideración, el trabajo plantea una apertura al análisis y construcción de nuevos indicadores que permitan evaluar la complejidad del periurbano y las necesidades de la población que lo habita.

El análisis de autocorrelación espacial se manifiesta como un medio válido para conocer y comprobar la situación de cada unidad espacial en relación con otras entidades geográficas, y así advertir la configuración los procesos socioambientales en el territorio. Asimismo, su desagregación por medio del reconocimiento de patrones locales de identificación espacial (LISA), ofrece una manera objetiva, cuantificable y reconocible en el modelo cartográfico resultante, para captar el momento del proceso de valoración de la calidad territorial en que se encuentran los recortes del mosaico periurbano. Es así que se identificaron a algunos radios costeros y limítrofes con la ciudad de Mar del Plata como aquellos que se encuen- 
tran en la mejor situación de acuerdo al $\mathrm{ICH}$. Sin embargo, predominan valores no significativos en grandes áreas de la zona de estudio que responden a situaciones indefinidas, características de los espacios en transformación.

En definitiva, la elaboración del ICH y su posterior representación cartográfica y estudio espacial, propician el análisis de las diferencias territoriales y socioambientales en las condiciones de habitabilidad resaltando las áreas de mayor contraste. La aplicación de esta metodología reviste un gran interés en el estudio del periurbano de Mar del Plata ya que favorece la detección de aquellas zonas más críticas, conformándose en una herramienta útil para minimizar el riesgo que representa en la gestión la toma de decisiones sin reconocer las diferenciaciones internas de los espacios.

\section{Referencias bibliográficas}

ABALERON, C. A. Calidad de vida como categoría epistemológica. Área, Revista de reflexión en Arquitectura, diseño y urbanismo, Universidad Nacional de Buenos Aires, 1998, No 6, p. 1-13.

ADELL, G. Theories and Models of the Peri-Urban Interface: A Changing Conceptual Landscape, Ouput 1. Research Projet: Strategis Environmental Planning and Management for de Peri-Urban Interface. Londres: DPU, 1999.

ANSELIN, L. GeoDa 0.9.5-i Release Notes. Spatial Analysis Laboratory. Department of Agricultural and Consumer Economics, University of Illinois. Illinois: Urbana-Champaign, 2004.

BARSKY, A. El periurbano productivo, un espacio en constante transformación. Introducción al estado del debate, con referencias al caso de Buenos Aires. En: VII Coloquio Internacional de Geocrítica "Los agentes urbanos y las políticas sobre la ciudad" (Santiago, 24-27 de mayo de 2005). Santiago de Chile: PUC Chile - U. de BarceIona, 2005.

BEN ALTABEF, C. La cuestión de la identidad en las prácticas profesionales y la en- señanza en el campo de la arquitectura. En: Congreso Latinoamericano de Educación Superior en el siglo XXI (San Luis, Argentina, 18-20 de septiembre de 2003). San Luis: Facultad de Ciencias Humanas, Universidad Nacional de San Luis, Argentina, 2003.

BONAVENA, H. Uso de suelo y estructura urbana actual del Partido de General Pueyrredón y de la ciudad de Mar del Plata. Ponencia en el marco del Plan Estratégico para Mar del Plata, Municipio de General Pueyrredon. Mar del Plata: Municipio de Pueyrredon, 2002.

BOZZANO, H. Territorios reales, territorios pensados, territorios posibles: aportes para una teoría territorial del ambiente. Buenos Aires: Espacio Editorial, 2004.

BURMESTER, M. Un estudio de caso: La problemática ambiental en la Cuenca Frutohortícola de la ciudad de Mar del Plata, Partido de General Pueyrredón. Tesis de Maestría en Gestión Ambiental del Desarrollo Urbano. Mar del Plata: Centro de Investigaciones Ambientales, FAUD-UNMdP, 2004.

BURMESTER, M.; GARAMENDI, J.; OLSZEWSKI, A. y SAGUA, M. La perspectiva ambiental para la gestión diversificada de un espacio urbano - regional. $I+A$, investigación + acción, Facultad de Arquitectura, Urbanismo y Diseño, Universidad Nacional de Mar del Plata, 2003, Año 7, No 8, p. 27-41.

BUZAI, G. y BAXENDALE, C. Análisis socioespacial con Sistemas de Información Geográfica. Buenos Aires: Lugar Editorial, 2006.

CANTER, L. W. Manual de evaluación de impacto ambiental. Técnicas para la elaboración de estudios de impacto. Colombia: Universidad de Oklahoma, Editorial McGraw-Hill, traducido de la segunda edición en inglés "Environmental Impact Assessment"; 1996, Editorial McGraw-Hill, USA, 1998.

CASTRO, M. E. Habitabilidad, medio ambiente y ciudad. En: $2^{\circ}$ Congreso Latinoamericano: El habitar, una orientación para la investigación proyectual (Buenos Aires, 6- 
9 de octubre de 1999). Buenos Aires: Universidad de Buenos Aires - Universidad Autónoma Metropolitana de México, 1999.

CEHAP. Vivienda y hábitat, claves en el tejido de ciudades: diagnóstico, líneas estratégicas y proyectos estratégicos. Medellín: Centro de Estudios del Hábitat Popular, Universidad Nacional de Colombia, Facultad de Arquitectura, 1998.

CELEMÍN, J. P. El estudio de la calidad de vida ambiental: definiciones conceptuales, elaboración de índices y su aplicación en la ciudad de Mar del Plata, Argentina. Revista Hologramática, 2007, Año IV, Vol. 1, No 7, p. 71-98.

CONESA FERNÁNDEZ-VÍTORA, V. Guía metodológica para la evaluación de impacto ambiental. Tercera Edición. Madrid: Ediciones Mundi-Prensa, 1997.

DEL RÍO, L. Funciones y disfunciones ambientales en la zona periurbana. Mar del Plata: Centro de Investigaciones Ambientales, FAUD-UNMdP, texto correspondiente a cátedra de la Maestría en Gestión Ambiental del Desarrollo Urbano, 2002.

DI PACE, M. (Dir.). Ecología de la ciudad. Buenos Aires: Editorial PrometeoUNGS, 2004.

ECHECHURI, H.; FERRARO, R., y BENGOA, G. Evaluación de Impacto Ambiental, entre el saber y la práctica. Buenos Aires: Centro de Investigaciones Ambientales, FAUD-UNMdP y Espacio Editorial, 2002.

ECHEVERRÍA, M. Usos del término Hábitat. Bogotá: Conferencia Maestría en Hábitat Universidad Nacional, 2002.

FERES, J.C. y MANCERO, X. El método de las Necesidades Básicas Insatisfechas (NBI) y sus aplicaciones en América Latina. Santiago de Chile: CEPAL, División de Estadística y Proyecciones Económicas, Serie de estudios estadísticos y prospectivos $N^{\circ} 7,2001$.

FERNÁNDEZ, R.; ALLEN, A.; BURMESTER, M.; MALVARES MÍGUEZ, M.; NAVARRO, L.; OLSZEWSKI, A. y SAGUA, M. Territorio, Sociedad y Desarrollo Sustentable.
Estudios de Sustentabilidad Ambiental Urbana. Buenos Aires: Espacio Editorial, Centro de Investigaciones Ambientales, FAUD, UNMdP, 1999.

FERNÁNDEZ，R.; SAGUA, M.; NAVARRO, L.; OLSZEWSKI, A.; BURMESTER, M.; MALVARES MÍGUEZ, M.; ALLEN, A. y GOYENECHE, H. El Observatorio Ambiental. Mar del Plata: Editorial Universitaria, 1998.

FERRARO, R. y ZULAICA, L. Sectorización del sistema periurbano de Mar del Plata, siguiendo criterios ambientales. En: U. MAYOR DE SAN SIMÓN. Congreso Internacional sobre desarrollo, medio ambiente $y$ recursos naturales, sostenibilidad a múltiples niveles y escalas. Cochabanba: Universidad Mayor de San Simón, Bolivia, 2007, № 3 .

GALLOPÍN, G. Sostenibilidad y DesarroIlo Sostenible: un enfoque sistémico. Santiago de Chile: CEPAL, Serie Medio Ambiente, División de Desarrollo Sostenible y Asentamientos Humanos, 2003, No 64 .

GARAY, A. Gestión ambiental de infraestructura y servicios urbanos. Mar del Plata: Centro de Investigaciones Ambientales, FAUD-UNMdP, texto correspondiente a la cátedra de la Maestría en Gestión Ambiental del Desarrollo Urbano, 1999.

GARCÍA, M. C. y VELÁZQUEZ, G. Percepción y medición de la calidad de vida en Tandil. En: VELÁZQUEZ, G. y GARCíA, M. C. Calidad de Vida Urbana: aportes para su estudio en Latinoamérica. Tandil: Centro de Investigaciones Geográficas, FCH, UNCPBA, 1999, p. 99-131.

GRUPO DE INVESTIGACIONES EN DESARROLLO SOCIAL (GIDES). Perspectivas del desarrollo comunitario y la calidad de vida en Cartagena: estudio de caso en los barrios La Central, El Milagro y San José de Los Campanos - Zona Sur Occidental. Cartagena: Centro de Investigaciones Cartagena, Universidad de San Buenaventura, 2003.

GÓMEZ OREA, D. Evaluación de Impacto Ambiental; un instrumento preventivo para la gestión ambiental. Madrid: Ediciones Mundi-Prensa y Editorial Agrícola Española S. A., 1999. 
GORDILLO BEDOYA, F. El hábitat: mutaciones en la ciudad y el territorio. Tabula Rasa, 2005, No 3, p. 137-149

GUIMARÃES, R. Tierra de sombras: desafíos de la sustentabilidad y del desarrollo territorial y local ante la globalización corporativa. Santiago de Chile: CEPAL, Serie Medio Ambiente, División de Desarrollo Sostenible y Asentamientos Humanos, 2003, $\mathrm{N}^{\circ} 67$.

HUBER, E. y GUÉRIN, M. A. Los cambios en las dimensiones semánticas de habitar. En: GIORDANO L. y D' ANGELI, L. (Eds.). El habitar, una orientación para la investigación proyectual. Buenos Aires: Universidad de Buenos Aires - Universidad Autónoma Metropolinana de México, Unidad Sochimilco, 2001, p. 347-353. Disponible en Internet: http://miguelguerin.com.ar/trabajosdeinvestigacion/loscambiosenlas dimensionessemanticas.pdf

Instituto Nacional de Estadísticas y Censos (INDEC). Censo Nacional de Población, Hogares y Viviendas. Buenos Aires: Instituto Nacional de Estadísticas y Censos, 2001. Disponible en Internet: www.indec. mecon.ar

LANDÁZURI ORTIZ, A. y MERCADO DOMÉNECH, S. Algunos factores físicos y psicológicos relacionados con la habitabilidad interna de la vivienda. Medio Ambiente y Comportamiento Humano, 2004, Vol. 5, $N^{\circ} 1-2$, p. 89-113. Disponible en Internet: http://webpages.ull.es/users/mach/PDFS/ Vol5_1y2/NOL_5_1y2_e.pdf

LUCERO, P. Población y poblamiento del Partido de General Pueyrredón: la combinación entre tiempo y espacio en la sociogeografía local. En: VELÁZQUEZ, G.; LUCERO, P. y MANTOBANI, J. Nuestra Geografía Local: población, urbanización y transformaciones socioterritoriales en el Partido de General Pueyrredón, Argentina, 1975-2000. Mar del Plata: Grupo de Estudios Sobre Población y Territorio, Departamento de Geografía, Facultad de Humanidades, UNMdP, 2004, p. 37-76.

LUCERO, P. y CELEMÍN, J. P. La calidad de vida de la población en la determinación de la calidad territorial. Un estudio de autocorrelación espacial aplicado a la ciudad de Mar del Plata. GeoFocus (Artículos), N ${ }^{\circ} 8$, 2008, p. 94-114. Disponible en Internet: http://geofocus.rediris.es/articulos 2008.html

LUCERO, P.; RIVIERE, I.; MIKKELSEN, C. y SABUDA, F. Brechas socioterritoriales vinculadas con la calidad de vida de los habitantes de Mar del Plata en los inicios del siglo XXI. En: VELAZQUEZ, G. y GÓMEZ LENDE, S. (Comp.). Desigualdad y Calidad de Vida en la Argentina (1991-2001). Aportes empíricos y metodológicos. Buenos $\mathrm{Ai}$ res: Centro de Investigaciones Geográficas, FCH, UNCPBA, Editorial Reun, 2005, p. 319-360.

MARINELLI, C.; TORCIDA, S.; CEPEDA, R.; GARCÍA, M. y VELÁZQUEZ, G. Un procedimiento alternativo para la selección estadística de variables de calidad de vida. En: VELÁZQUEZ, G. y GARCÍA, M. C. Calidad de Vida Urbana: aportes para su estudio en Latinoamérica. Tandil: Centro de Investigaciones Geográficas, FCH, UNCPBA, 1999, p. 133-141.

MIKKELSEN, C. Ampliando el estudio de la calidad de vida hacia el espacio rural. El caso del Partido de General Pueyrredón, Argentina. Revista Hologramática, Facultad de Ciencias Sociales, UNLZ, 2007, Año IV, Vol. 4, No 6, p. 25-48.

MORELLO, J. Funciones del sistema periurbano: el caso de Buenos Aires. Mar del Plata: Centro de Investigaciones Ambientales, FAUD-UNMdP, texto correspondiente a la cátedra de la Maestría en Gestión Ambiental del Desarrollo Urbano, 2000.

MORENO, C. Relaciones entre vivienda, ambiente y hábitat. Medellín: Universidad Nacional de Colombia, 2002.

MORENO JIMÉMEZ, A. Sistemas y Análisis de la Información Geográfica. Madrid: Ra-Ma Editorial, 2006.

OLAVE FARÍAS, D. (Ed.). Ciudades intermedias y calidad de vida: conceptos básicos Departamento de ciencias sociales. Santiago de Chile: Universidad del Bío-Bío, Facultad de Educación y Humanidades, 2001. 
RIVIERE, I. y CELEMÍN, J. P. Los recuperadores del predio de disposición final de residuos sólidos en la ciudad de Mar del Plata. En: 8vo. Encuentro Internacional Humboldt. (Colón, Argentina, 26-28 de abril de 2006). Colón: Centro de Estudios Alexander Von Humboldt, 2006.

RIVIERE, I.; LUCERO, P.; MIKKELSEN, C. y SABUDA, F. Disparidades Territoriales en la Calidad de Vida de la población de Mar del Plata, en los tiempos de la Argentina Globalizada. En: Seminario Internacional de Población y Sociedad (Salta, 8-10 de junio de 2005). Salta: GREDES, 2005.

RUEDA, S. Habitabilidad y calidad de vida; ciudades para un futuro sostenible. Documentos La construcción de la ciudad sostenible, 1997. Disponible en Internet: http://habitat.aq.upm.es/cs/p2/a005.html

RUEDA, S. Modelos e indicadores para ciudades más sostenibles. Cataluña: Fundació Fòrum Ambiental, 1999. Disponible en Internet: http://www.forumambiental.org/ pdf/huella.pdf

SAGUA, M. Dinámica sociodemográfica y ambiente al interior del Partido de General Pueyrredón. En: VELÁZQUEZ, G.; LUCERO, P. y MANTOBANI, J. Nuestra Geografía Local: población, urbanización y transformaciones socioterritoriales en el Partido de General Pueyrredón, Argentina, 1975-2000. Mar del Plata: Grupo de Estudios Sobre Población y Territorio, Departamento de Geo- grafía - Facultad de Humanidades, UNMdP, 2004, p. 129-165.

URRUZOLA, J. P. Pensar el territorio. Revista Relaciones, Serie Ser Urbano, 2006, No 269. Disponible en Internet: http:// fp.chasque.net/ relacion/0610/index.htm

VELÁZQUEZ, A. y GÓMEZ LENDE, S. Población y calidad de Vida en la Argentina (1991-2001), La fragmentación de la sociedad y el territorio. En: VELÁZQUEZ, G. y GÓMEZ LENDE, S. Desigualdad y Calidad de Vida en la Argentina (1991-2001). Aportes empíricos y metodológicos. Buenos Aires: Centro de Investigaciones Geográficas, $\mathrm{FCH}$, UNCPBA, Editorial Reun, 2005, p. 199-240.

VELÁZQUEZ, G. Geografía, calidad de Vida y Fragmentación en la Geografía de los noventa. Análisis regional y departamental utilizando SIG's. Tandil: Centro de Investigaciones Geográficas, FCH, UNCPBA, 2001.

VILALTA Y PERDOMO, C. Cómo enseñar autocorrelación espacial. Economía, Sociedad, y Territorio, 2005, Vol. V, No 1, p. 323-333.

ZULAICA, L. y FERRARO, R. El periurbano de Mar del Plata: complejidad territorial y dificultades para su ordenamiento. En: IV Seminario de Ordenamiento Territorial (Mendoza, 7-9 de noviembre de 2007). Mendoza: Instituto CIFOT, Facultad de Filosofía y Letras, Universidad Nacional de Cuyo, 2007. 